\title{
POLY (SODIUM ACRYLATE) HYDROGELS AS POTENTIAL pH-SENSITIVE SORBENTS FOR THE REMOVAL OF MODEL ORGANIC AND INORGANIC POLLUTANTS FROM WATER
}

\author{
V. BEKIARI ${ }^{1, *}$ \\ P. LIANOS ${ }^{2}$
}

${ }^{1}$ Department of Aquaculture and Fisheries Management Technological Educational Institute of Messolonghi 30200 Messolonghi, Greece

${ }^{2}$ Engineering Science Department University of Patras, GR-26504 Patras, Greece

Received: 06/05/10 Accepted: 30/08/10

*to whom all correspondence should be addressed: e-mail: mpekiari@teimes.gr

\section{ABSTRACT}

Anionic poly(sodium acrylate), PANa, hydrogels bearing negatively charged $-\mathrm{COO}^{-}$groups were used as sorbents for the removal of colored organic or inorganic model pollutants, namely Crystal Violet and $\mathrm{Cu}^{2+}$, from water. It was found that PANa hydrogels adsorb stronger $\mathrm{Cu}^{2+}$ ions than Crystal Violet, as a consequence of the formation of coordination complexes between the hydrogel and the metal ions, while just electrostatic interactions are the major driving forces for Crystal Violet. Furthermore, the influence of $\mathrm{pH}$, i.e. the neutralization degree $\mathrm{i}$ of the hydrogel, on its adsorption efficiency was investigated. It is shown that the adsorption of $\mathrm{Cu}^{2+}$ ions follows stoichiometry, namely one metal ion per two carboxylate anions. On the contrary, the adsorption of Crystal Violet is much lower than the charged stoichiometry.

KEYWORDS: poly (sodium acrylate), hydrogels, adsorption, ionization degree, $\mathrm{Cu}^{2+}$, Crystal Violet.

\section{INTRODUCTION}

The development of novel sorbents, able to remove organic or heavy metal pollutants from wastewaters, represents an intensive and popular research field during the last years. Thus, besides the classical activated carbon filters, different types of other materials have been proposed and developed, like organically modified synthetic porous silica (Bekiari and Lianos, 2006; Chu et al., 2008; Sayari et al., 2005), dendritic polymers (Arkas et al., 2003; Arkas et al., 2005) and hydrogels (Bekiari et al., 2008; Kofinas and Koussis, 2003). Hydrogels are three-dimensional chemically crosslinked polymer networks, able to swell in water, increasing substantially their original volume while keeping their integrity. These polymeric materials are promising for environmental applications, in particular, the waste water treatment (Bajpai and Johnson, 2005; Bekiari et al., 2008; Chauhan et al., 2006; Kasgoz et al., 2003; Kofinas and Koussis, 2003; Ray et al., 2007; Thivaios and Bokias, 2010). In fact, it is known that charged hydrogels are able to adsorb effectively from water oppositely charged organic and inorganic pollutants. Thus, numerous studies have been devoted to the adsorption of cationic dyes, used as model pollutants, by anionic hydrogels (Jeon et al., 2008; Paulino et al., 2006; Solpan et al., 2003; Tang et al., 2009) containing weakly acidic or strongly charged groups.

Cross-linked poly (sodium acrylate), PANa (Figure 1), is a synthetic hydrophilic polymer bearing carboxylate anions along the macromolecular chain, (Molyneux, 1984; Bekturov and Bakauova, 1986). This material probably represents the simplest case of an anionic hydrogel, able to adsorb cationic species, like positively charged pollutants or organic dyes (Mak and Chen, 2004) and higher valence metal ion (Rivas et al., 2003). The combination of electrostatic attractions with hydrophobic interactions is usually the driving force in the case of cationic organic species, while polymer/metal ion coordination complexes are often formed when higher valency metal ions are involved. For 
instance, when PANa is mixed in aqueous solution with $\mathrm{Cu}^{2+}$ ions, the coordination of the carboxylate anions with the metal ion leads to the formation of a PANa-Cu ${ }^{2+}$ complex (Francois et al., 1997; Gregor et al., 1955; Ikeda et al., 1998; Mun et al., 2003; Rivas et al., 2005; Yokoi et al., 1986). For simplicity reasons, the PANa-Cu${ }^{2+}$ complexation can be described by eq. (1):

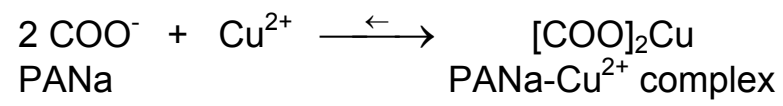

suggesting that complexation involves two carboxylate groups per $\mathrm{Cu}^{2+}$ ion (Francois et al., 1997; Kislenko and Verlinskaya, 2001; Mandel and Leyte, 1964; Morawetz, 1955).

It should be stressed that in all cases, the interactions of PANa with positively charged species can be modulated by $\mathrm{pH}$ changes. Indeed, PANa turns to the uncharged poly(acrylic acid), PAA, form by decreasing $\mathrm{pH}$, as a consequence of the weak acidic character of the carboxylic groups of the polymer in aqueous solution. This effect is described in Eq. (2):<smiles>[CH+]C(CC)C(=O)[O-]</smiles>

\section{PANa}

PAA

The influence of $\mathrm{pH}$, namely the degree of ionization of PAA, on the adsorption abilities of the respective hydrogels is systematically investigated in the present work. Two colored species, namely $\mathrm{Cu}^{2+}$ ions and Crystal Violet (Figure 1), were selected as model inorganic and organic pollutants. Thus, the remaining pollutant in the aqueous solution after adsorption could be monitored by UV-vis absorption spectrophotometry.

\section{EXPERIMENTAL}

\subsection{Materials}

Acrylic acid (AA), N,N,N,N'tetramethylethylenediamine (TEMED) and methylene bisacrylamide (BIS) were purchased from Aldrich, while ammonium persulfate (APS) was a Serva product.

Crystal Violet (CV) and $\mathrm{CuSO}_{4} \cdot 6 \mathrm{H}_{2} \mathrm{O}$ were purchased from Aldrich. All reagents were used as received.

Water was purified by means of a TKA Smart2Pure apparatus.

\subsection{Synthesis of the hydrogels}

The PANa hydrogel were synthesized according to the following procedure: $0.05 \mathrm{~mol}$ AA and 0.001 mol BIS (crosslinker, 2 mol\% over the total monomer concentration) were dissolved in $40 \mathrm{~mL}$ ultrapure water under stirring at room temperature. The $\mathrm{pH}$ of the solution was adjusted to $\mathrm{pH}=5-6$ by addition of adequate volume of a $\mathrm{NaOH} 1 \mathrm{M}$ solution. After deoxygenation of the solution by $\mathrm{N}_{2}$ bubbling for $30 \mathrm{~min}, 0.1 \mathrm{~g}$ APS (dissolved in $2 \mathrm{~mL}$ ultrapure water) and 3 drops of TEMED were added. The formation of the gel was almost immediate. The reaction was left to proceed for $24 \mathrm{~h}$ and, then, the gel was fully neutralized to PANa form by adding an excess of the $\mathrm{NaOH} 1 \mathrm{M}$ solution $(\mathrm{pH}=13)$. After $24 \mathrm{~h}$, the gel was immersed in ultrapure water. Water was decanted and renewed daily for 1 week. Finally, the purified swollen gel was cut into $\sim 2 \mathrm{~mL}$ pieces and water was removed by freeze-drying.

\subsection{Adsorption of ions from aqueous solutions}

The adsorption of ions was studied by the following procedure: $\sim 0.02 \mathrm{~g}$ of dry hydrogel was introduced in $10 \mathrm{~mL}$ of aqueous solution of $\mathrm{Cu}^{2+}$ or in $100 \mathrm{~mL}$ of aqueous solution of Crystal Violet and was left to adsorb for $24 \mathrm{~h}$. Then, the gel was removed from the solution and the remaining ions (i.e. $\mathrm{CV}$ and $\mathrm{Cu}^{2+}$ ) were monitored by UV-Vis absorption spectrophotometry. In each case, the experiments were done for five different initial concentrations of cations. The studied concentration range was from $1 \mu \mathrm{M}$ to $1 \mathrm{mM}$ for Crystal Violet and from $2.5 \mathrm{mM}$ to $25 \mathrm{mM}$ for $\mathrm{Cu}^{2+}$ ions. In particular cases, prior adsorption experiments, the ionization degree of the hydrogel was adjusted by adding the appropriate amount of $\mathrm{HCl}$. 


\subsection{Methods}

UV-vis absorption measurements were performed with a Shimadzu UV 1800 spectrophotometer. Langmuir isotherms were obtained by keeping constant the quantity of the adsorbing material and varying the initial concentration of the ions in the solution. The concentration of the solute was calculated from the respective calibration curves for both ions studied.<smiles>CN(C)c1ccc(C(=C2C=CC(=[NH2+])C=C2)c2ccc(N(C)C)cc2)cc1</smiles>

Crystal Violet

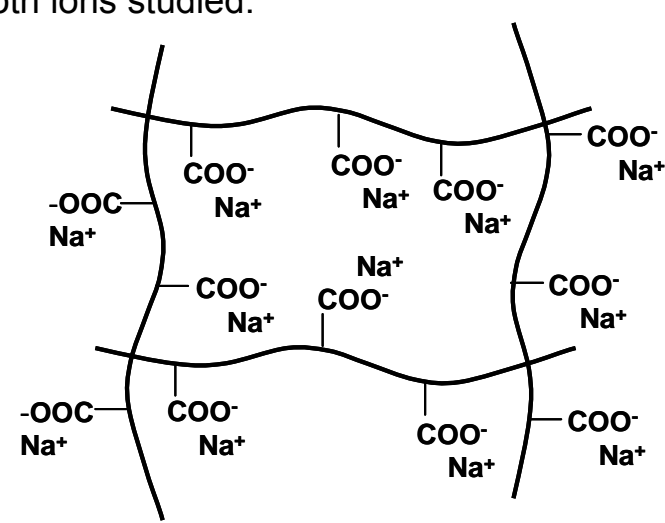

PANa

Figure 1. Chemical structure of the dye and the schematic depiction of the PANa hydrogel used in the present work

\section{RESULTS AND DISCUSSION}

As previously discussed, the prime criterion for the selection of the above model pollutants is their substantial light absorption in the UV-Vis spectral range. The exploitation of the obtained UV-vis spectra is demonstrated in Figures 2 and 3, showing characteristic examples of the adsorption experiments performed using $\mathrm{Cu}^{2+}$ ions and Crystal Violet, respectively.

The aqueous $\mathrm{CuSO}_{4}$ solution is blue-colored, as a result of a large absorption band in the visible region, centered at $\sim 820 \mathrm{~nm}$. After treatment with PANa hydrogel, absorbance of the solution was hardly detectable, indicating that most $\mathrm{Cu}^{2+}$ ions have been removed from water under these experimental conditions. Instead, the initially transparent PANa hydrogel was then strongly-colored (inset of Figure 2), providing a direct visual evidence for the strong adsorbing capacity of the hydrogel towards $\mathrm{Cu}^{2+}$ ions. The strong green-blue color of the hydrogel results both form the increased concentration of $\mathrm{Cu}^{2+}$ ions in the volume of the hydrogel and from the polymer-metal ion complexation. In fact, it is known that the extinction coefficient is greatly enhanced upon complexation of PANa with $\mathrm{Cu}^{2+}$ ions, while the absorption band is shifted towards $\sim 720 \mathrm{~nm}$ (Francois et al., 1997).

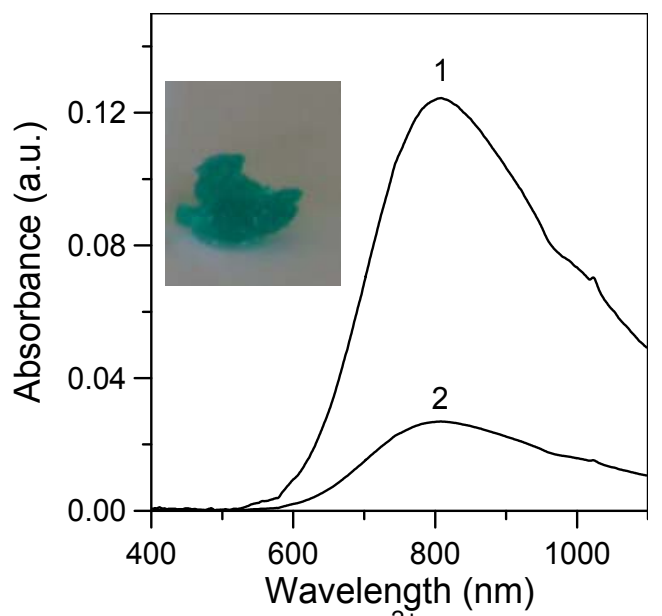

Figure 2. Absorption spectra of aqueous $10 \mathrm{mM} \mathrm{Cu}^{2+}$ ions before (1) and after (2) equilibration with the PANa hydrogel. The appearance of the hydrogel at the end of the adsorption experiment is shown in the inset 


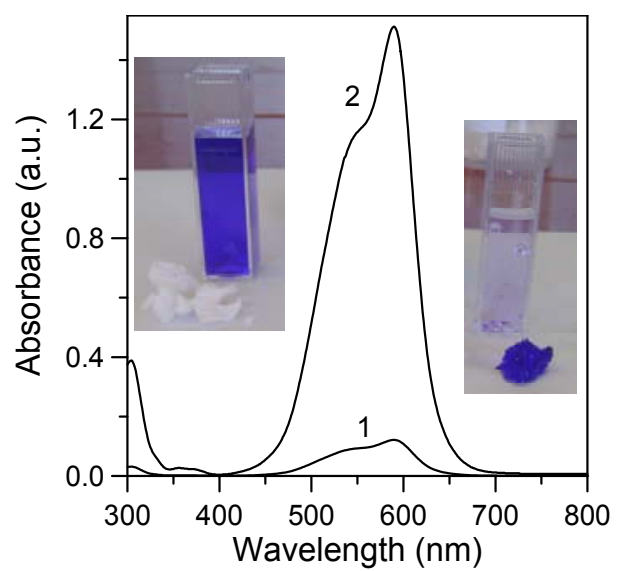

Figure 3. Absorption spectra of aqueous $0.02 \mathrm{mM}$ Crystal Violet before (1) and after (2) equilibration with the PANa hydrogel. The appearance of the aqueous solution and the hydrogel initially and at the end of the adsorption experiment are shown in the left and right inset, respectively

Similar adsorption experiments were performed using Crystal Violet. A characteristic example is given in Figure 3 which also shows the appearance of the aqueous solution and the hydrogel before (left inset) and after (right inset) equilibration with the solution of the dye.). Due to the high extinction coefficient of $\mathrm{CV}$ compared to $\mathrm{Cu}^{2+}$, the studied concentrations for the organic dye were lower than those studied for the inorganic ion (see Figs 2 and 3). The dye solution is strongly colored blue-violet before equilibration, while the characteristic absorption band centered at $\sim 600 \mathrm{~nm}$ is monitored in the visible region. After equilibration with the PANa hydrogel, this absorption band was much weaker, in agreement with the discoloration of the aqueous solution, which can be visually detected. In contrast, the PANa hydrogel was now strongly colored, showing that most of the dye was adsorbed under the present experimental conditions.

The anionic character of the hydrogel is expected to control its adsorbing ability towards oppositely charged species, such as $\mathrm{Cu}^{2+}$ ions or Crystal Violet, as mentioned in the Introduction section. For this reason, a systematic adsorption study was undertaken as a function of the charge density of the PANa hydrogel, as expressed by the neutralization degree, i,

$$
i=\frac{n_{\mathrm{COO}^{-}}}{n_{\mathrm{COO}^{-}}+n_{\mathrm{COOH}}}
$$

where $\mathrm{n}_{\mathrm{COO}}$ and $\mathrm{n}_{\mathrm{COOH}}$ are the moles of the carboxylate anions and carboxylic groups, respectively, contained in the hydrogel. Since the hydrogels were initially obtained under the fully charged PANa form ( $i=1)$, the neutralization degree $\mathrm{i}$ was adjusted by adding the appropriate quantity of an aqueous $\mathrm{HCl}$ solution, following Eq. (2). Samples with $\mathrm{i} \geq 0.25$ were investigated, in order to assure a prominent ionic character for the hydrogel.

The Langmuir isotherms for the adsorption of $\mathrm{Cu}^{2+}$ ions and Crystal Violet from the aqueous solution by the hydrogels with ionization degrees covering the range $0.25 \leq i \leq 1$ are presented in Figures 4 and 5 . In these representations, the quantity $Q_{e}$ expresses the mmoles of the adsorbed ion per gram of the adsorbent and it is calculated by the equation (Bekiari and Lianos, 2006; Sayari et al., 2005):

$Q e=\left(C_{0}-C_{e}\right) \frac{V}{m}$

where $\mathrm{C}_{0}$ is the initial concentration of the studied substance (in $\mathrm{mmol} \mathrm{L}^{-1}$ ), $\mathrm{V}$ is the volume of the solution used for the adsorption (in $\mathrm{L}$ ) and $\mathrm{m}$ is the mass of the dry PANa hydrogel (in $\mathrm{g}$ ) weighed under the fully neutralized PANa form. $\mathrm{C}_{\mathrm{e}}$ is the concentration of the ions remaining in solution, and it is determined by the calibration curves constructed at $805 \mathrm{~nm}$ and $590 \mathrm{~nm} \mathrm{for} \mathrm{Cu}^{2+}$ ions and Crystal Violet, respectively.

As seen in Figures 4 and 5, the investigation with Crystal Violet was possible for very low dye concentrations, due to the much higher extinction coefficient of Crystal Violet as compared to that of $\mathrm{Cu}^{2+}$ ions. For both solutes, it was observed that $Q_{e}$ initially increases with $C_{e}$ but it soon attained the maximum value $Q_{e, \text { max }}$. This shows that the hydrogel is saturated with the adsorbed species and it can not adsorb anymore $\mathrm{Cu}^{2+}$ ions or Crystal Violet. In fact, although it is not always very clear, it seems that $Q_{e, m a x}$ is attained at lower $C_{e}$ values as i decreases from 1 down to 0.25 . Such a behavior 
could be explained by the respective decrease of interacting units, namely carboxylate anions as $\mathrm{i}$ decreases. This is also the origin of the substantial decrease of $Q_{e, m a x}$ as i decreases, observed for both solutes. Moreover, comparing the two Figures, it is noteworthy that the $Q_{e, m a x}$ values attained for Crystal Violet are significantly lower than the corresponding values obtained for $\mathrm{Cu}^{2+}$ ions at the same ionization degree $\mathrm{i}$.

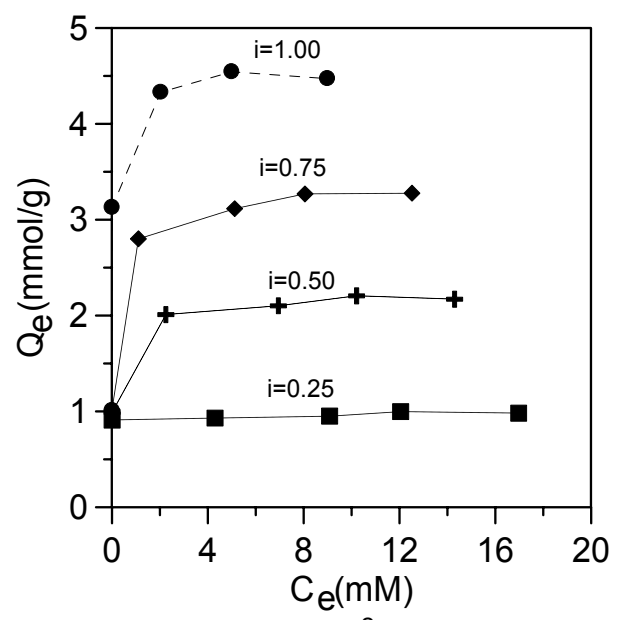

Figure 4. Plot of the quantity $\mathrm{Q}_{e}$ of the adsorbed $\mathrm{Cu}^{2+}$ as a function of the concentration $\mathrm{C}_{e}$ of the cation remaining in solution for several neutralization degrees (i) of the hydrogel

To better quantify the aforementioned observations, the dependence of $\mathrm{Q}_{\mathrm{e} \text {, max }}$ attained for $\mathrm{Cu}^{2+}$ ions on the neutralization degree $\mathrm{i}$ of the hydrogels is compared in Figure 6 with the respective results obtained with Crystal Violet. The solid line in Figure 6 represents the charge content $f$ of the hydrogels (in mmoles of carboxylate anions per $\mathrm{g}$ of dry hydrogel), determined as

$f=\frac{i \times(\mathrm{m} / \mathrm{g} / \mathrm{g} / \mathrm{mol})}{\mathrm{m}} \times 1000 \frac{\mathrm{mmol}}{\mathrm{mol}}=10.6 \times i \frac{\mathrm{mmol}}{\mathrm{g}}$

where $94 \mathrm{~g} \mathrm{~mol}^{-1}$ is the molar mass of the repeating sodium acrylate unit.

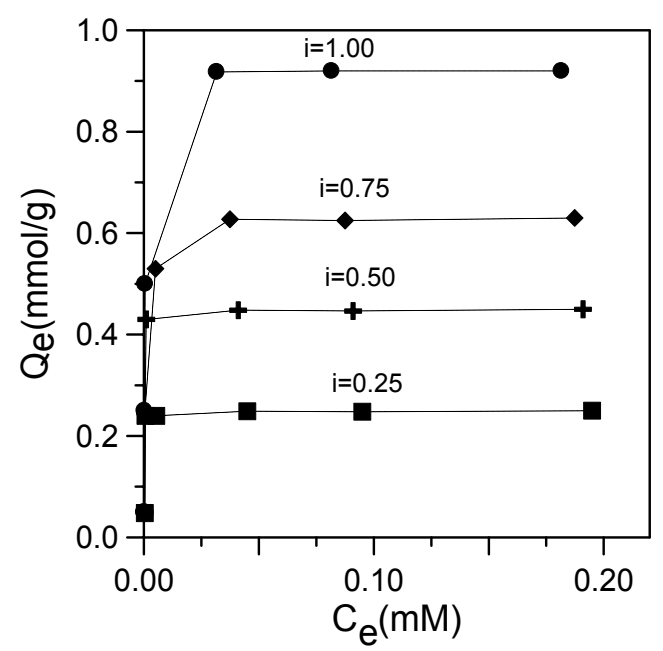

Figure 5. Plot of the quantity $Q_{e}$ of the adsorbed Crystal Violet as a function of the concentration $\mathrm{C}_{\mathrm{e}}$ of the dye remaining in solution, for several neutralization degrees (i) of the hydrogel

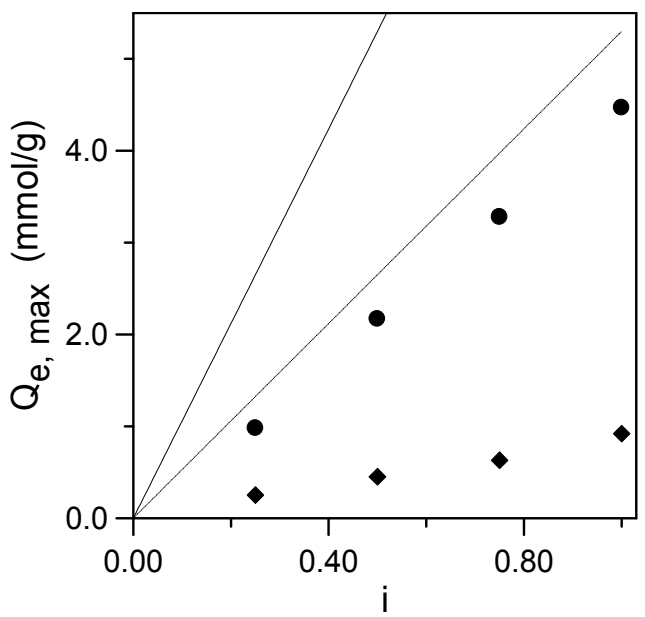

Figure 6. Variation of $\mathrm{Q}_{\mathrm{e}, \max }$ with neutralization degree (i) of the hydrogel for the adsorption of $\mathrm{Cu}^{2+}$ ions $(\bullet)$ and Crystal Violet $(\bullet)$. The solid and the dashed line represents the charge density and the half-charge density of the hydrogel, respectively

It is clear from Figure 6 that $Q_{e, \max }$ is 0 when the hydrogel is uncharged ( $i$ tends to 0 ) and increases linearly with $\mathrm{i}$ both for $\mathrm{Cu}^{2+}$ ions and Crystal Violet. Meorover, the maximum adsorbed quantity of $\mathrm{Cu}^{2+}$ is much higher than that of Crystal Violet for the same neutralization degree $\mathrm{i}$. Thus, the highest 
$Q_{e, \text { max }}$ value at $i=1$ is $\sim 4.5 \mathrm{mmol} \mathrm{g}^{-1}$ for Cu2+ ions and about the one fifth $\left(\sim 0.9 \mathrm{mmol} \mathrm{g}^{-1}\right)$ for Crystal Violet. This suggests that the adsorbing ability of the above hydrogels is much stronger towards $\mathrm{Cu}^{2+}$ ions with respect to the organic dye. In fact, this behavior is not unexpected, since the driving force in the latter case, namely electrostatic interactions (possibly corroborated by hydrophobic interactions), is rather weak, as compared to the coordination complexes formed in the case of $\mathrm{Cu}^{2+}$ ions. Finally, It is interesting to note that the $\mathrm{Q}_{e, \max }$ values obtained for $\mathrm{Cu}^{2+}$ ions are very close (somewhat lower) to the half-charged density of the hydrogels studied at each neutralization degree $\mathrm{i}$, represented by the dotted line in Figure 6 . This offers an additional indirect evidence that the stoichiometry of the coordination complex formed is two carboxylate anions per $\mathrm{Cu}^{2+}$ ion, as it was also shown when linear PANa chains were used instead of hydrogels (latridi et al., 2008).

As a final step in our study, we attempted an analysis of the obtained Langmuir isotherms using the following equation (Arkas et al., 2003)

$\frac{C_{e}}{Q_{e}}=\frac{1}{K_{L}}+\left(\frac{\alpha_{L}}{K_{L}}\right) C_{e}$

where $K_{L}$ is the binding constant expressed in $\mathrm{L} \mathrm{g}^{-1}$ and $\alpha_{\mathrm{L}}$ is the Langmuir constant. The variation of $\mathrm{C}_{e} / \mathrm{Q}_{\mathrm{e}}$ with $\mathrm{C}_{\mathrm{e}}$ is plotted in Figures 7 and 8 for the results shown in Figures 4 and 5 , respectively. In all cases, straight lines are obtained, indicating that adsorption obeys the Langmuir model. Moreover, the intercepts of all lines are close to 0 . This suggests that the binding constants $\mathrm{K}_{\mathrm{L}}$ are very large for both adsorbed species, in agreement with previously reported results where crosslinked copolymers of sodium acrylate with N,N-dimethylacrylamide were investigated (Bekiari et al., 2008). However, it is not possible to reliably determine the accurate values of $K_{L}$ from the present experimental data, since a reliable determination of $\mathrm{C}_{e}$ in very dilute solutions is needed. To this end, atomic absorption spectroscopy could be useful, for instance, in the case of $\mathrm{Cu}^{2+}$ ions. Such an investigation is now under way in our lab.

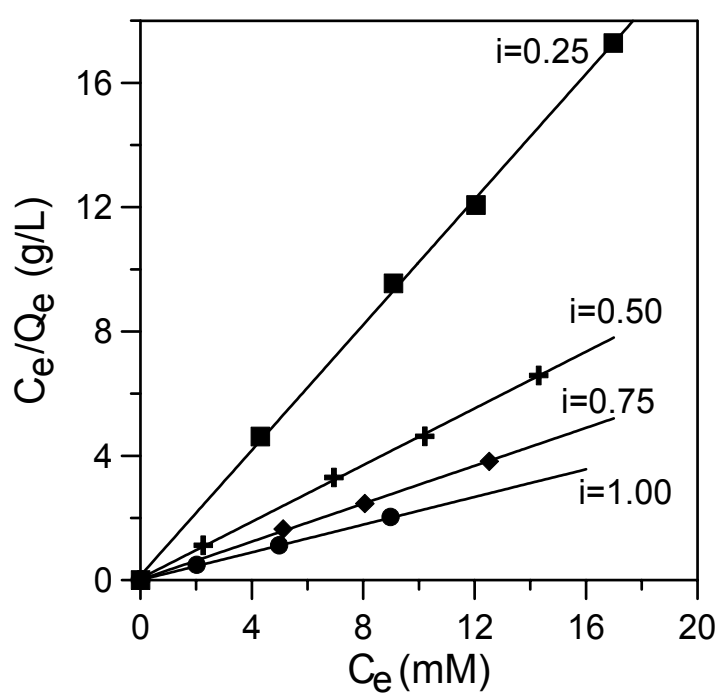

Figure 7. Plot of the quantity $\mathrm{C}_{\mathrm{e}} / \mathrm{Q}_{\mathrm{e}}$ of the adsorbed $\mathrm{Cu}^{2+}$ as a function of the concentration $\mathrm{C}_{e}$ of the cation remaining in solution for several neutralization degrees (i) of the hydrogel

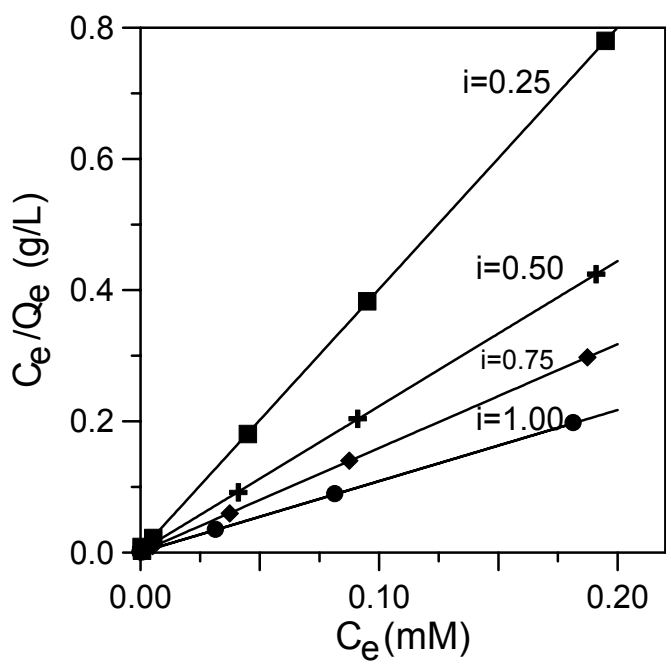

Figure 8 . Plot of the quantity $\mathrm{C}_{\mathrm{e}} / \mathrm{Q}_{\mathrm{e}}$ of the adsorbed Crystal Violet as a function of the concentration $\mathrm{C}_{e}$ of the dye remaining in solution for several neutralization degrees (i) of the hydrogel

\section{CONCLUSIONS}

The possibility of using poly (sodium acrylate), PANa, hydrogels as sorbents for removing oppositely charged pollutants from aqueous systems was investigated in the present study. To this purpose, $\mathrm{Cu}^{2+}$ ions and Crystal Violet were selected as model inorganic and organic pollutants. It was found that PANa hydrogels are efficient sorbents for both pollutants. Moreover, the adsorption capacity of PANa hydrogels is much larger towards $\mathrm{Cu}^{2+}$ ions, as compared to Crystal Violet. This is attributed to the formation of coordination complexes in the first case, whereas electrostatic binding is the major driving force in the case of Crystal Violet. 
Special attention was paid on the influence of $\mathrm{pH}$, i.e. the neutralization degree $\mathrm{i}$ of the hydrogel, on its adsorption efficiency. Although it was not directly studied here, a clear tendency of complete desorption of both species is evidenced as i tends to 0 . A related quantitative investigation is now under way. This behavior is interesting, since it could be possibly exploited for the recovery of the pollutants and the regeneration of the sorbent.

\section{REFERENCES}

Arkas M.,Tsiourvas D. and Paleos C. (2003) Functional Dendrimeric "Nanosponges" for the Removal of Polycyclic Aromatic Hydrocarbons from Water, Chem. Mater., 15, 2844-2847.

Arkas M.,Tsiourvas D. and Paleos C. (2005) Organosilicon Dendritic Networks in Porous Ceramics for Water Purification, Chem. Mater., 17, 3439-3444.

Bajpai S.K. and Johnson S. (2005) Superabsorbent hydrogels for removal of divalent toxic ions. Part I: Synthesis and swelling characterization, React. Funct. Polym., 62, 271-283.

Bekiari V. and Lianos P. (2006) Ureasil Gels as a Highly Efficient Adsorbent for Water Purification, Chem. Mater., 18, 4142-4146.

Bekiari V., Sotiropoulou M., Bokias G. and Lianos P. (2008) Use of poly(N,N, dimethylacrylamide-cosodium acrylate) hydrogel to extract cationic dyes and metals from water,Colloids Surf. A, 312, 214218.

Bekturov E.A. and Bakauova Z.Kh. (1986) Synthetic Water-Soluble Polymers in Solution, Huethig and Wepf.

Chauhan G.S., Singh B., Sharma R.K., Verma M., Jaswal S.C. and Sharma R. (2006) Use of biopolymers and acrylamide-based hydrogels for sorption of $\mathrm{Cu}^{2+}, \mathrm{Fe}^{2+}$ and $\mathrm{Cr}^{6+}$ ions from their aqueous solutions, Desalination, 197, 75-81.

Chu C-C., Ueno N. and Imae T. (2008) Solid-Phase Synthesis of Amphiphilic Dendron-Surface Modified Silica Particles and Their Application Toward Water Purification, Chem. Mater., 20, 2669-2676.

Francois J., Heitz C. and Mestdagh M.M. (1997) Spectroscopic study (u.v.-visible and electron paramagnetic resonance) of the interactions between synthetic polycarboxylates and copper ions, Polymer, 38, 5321-5332.

Gregor H.P., Luttinger L.B. and Loebl E. (1955) Metal-Polyelectrolyte Complexes. I. The Polyacrylic Acid-Copper Complex, J. Phys. Chem., 59, 34-39.

latridi Z., Bokias G. and Kallitsis J.K. (2008) Physicochemical study of the complexation of poly(acrylic acid) with $\mathrm{Cu}^{2+}$ ions in water, J. Appl. Polym. Sci., 108, 769-776.

Ikeda Y., Beer M., Schmidt M. and Huber K. (1998) $\mathrm{Ca}^{2+}$ and $\mathrm{Cu}^{2+}$ Induced Conformational Changes of Sodium Polymethacrylate in Dilute Aqueous Solution, Macromolecules, 31, 728-733.

Jeon Y.S., Lei J. and Kim J-H. (2008) Dye adsorption characteristics of alginate/polyaspartate hydrogels, J. Ind. Eng. Chem.,14, 726-731.

Kasgoz H., Ozgumus S. and Orbay M. (2003) Modified polyacrylamide hydrogels and their application in removal of heavy metal ions, Polymer, 44, 1785-1793.

Kislenko V.N. and Verlinskaya R.M. (2001) Peculiarities of Polyacrylic Acid Adsorption on Copper Oxide Powder, Colloid J., 63, 558-562.

Kofinas P. and Koussis D.R. (2003) Reactive Phosphorus Removal from Aquaculture and Poultry Productions Systems Using Polymeric Hydrogels, Environ. Sci. Technol., 37, 423-.427.

Mak S-Y. and Chen D-H. (2004) Fast adsorption of methylene blue on polyacrylic acid-bound iron oxide magnetic nanoparticles, Dyes and Pigments, 61, 93-98.

Mandel M. and Leyte J.C. (1964) Interaction of poly(methacrylic acid) and bivalent counterions. I, J. Polym. Sci. Part A, 2, 2883-2899.

Molyneux P. (1984) Water-Soluble Synthetic Polymers: Properties and Behavior Vol. II; CRC Press: Florida.

Morawetz H. (1955) The nature of copper (II) complexes with poly(acrylic acid) and poly(methacrylic acid), J. Polym. Sci., 17, 442-444.

Mun G.A., Nurkeeva Z.S., Khutoryanskiy V.V., Sarybayeva G.S. and Dubolazov A.V. (2003) pH-effects in the complex formation of polymers I. Interaction of poly(acrylic acid) with poly(acrylamide), Eur. Polym. J., 39, 1687-1691.

Paulino A.T., Guilherme M.R., Reis A.V., Campese G.M., Muniz E.C. and Nozaki J. (2006) Removal of methylene blue dye from an aqueous media using superabsorbent hydrogel supported on modified polysaccharide, J Colloid Interface Sci., 301, 55-62.

Ray S., Das A.K. and Banerjee A. (2007) pH-Responsive, Bolaamphiphile-Based Smart MetalloHydrogels as Potential Dye-Adsorbing Agents, Water Purifier, and Vitamin B12 Carrier, Chem. Mater., 19, 1633-1639. 
Rivas B.L., Pereira E.D. and Moreno-Villoslada I. (2003) Water-soluble polymer-metal ion interactions Prog. Polym. Sci., 28, 173-208.

Rivas B. L., Pooley S.A., Brovelli F., Pereira E., Basaez L., Osorio F., Moutet J. and Saint-Adam E. (2005) Synthesis and properties of polymer film modified electrodes to detect metal ions, J. Appl. Polym. Sci., 98, 1192-1197.

Sayari A., Hamoudi S. and Yang Y. (2005) Applications of Pore-Expanded Mesoporous Silica. 1. Removal of Heavy Metal Cations and Organic Pollutants from Wastewater, Chem. Mater., 17, 212216.

Solpan D., Duran S., Saraydin D. and Guven O. (2003) Adsorption of methyl violet in aqueous solutions by poly(acrylamide-co-acrylic acid) hydrogels, Rad. Phys. Chem., 66, 117-127.

Tang Q., Wu J., Sun H., Fan S., Hu D. and Lin J. (2009) Synthesis of polyacrylate/poly(ethylene glycol) hydrogel and its absorption properties for heavy metal ions and dye, Polymer Composites, 30,1183-1189.

Thivaios I. and Bokias G. (2010) Adsorption of nile red by poly(N-isopropylacrylamide) gels in binary water/tetrahydrofuran mixtures, J. Appl. Polym. Sci., 116, 1509-1514.

Yokoi H., Kawata S. and Iwaizumi M. (1986) Interaction modes between heavy metal ions and watersoluble polymers. 2. Spectroscopic and magnetic reexamination of the aqueous solutions of cupric ions and poly(acrylic acid), J. Am. Chem. Soc., 108, 3361-3365. 